\title{
Light scattering at textured back contacts for $n$-i-p thin-film silicon solar cells
}

K. Bittkau, W. Böttler, M. Ermes, V. Smirnov, and F. Finger

Citation: Journal of Applied Physics 111, 083101 (2012);

View online: https://doi.org/10.1063/1.3703572

View Table of Contents: http://aip.scitation.org/toc/jap/111/8

Published by the American Institute of Physics

\section{Articles you may be interested in}

Molecular beam epitaxial growth and characterization of $\operatorname{InSb}_{1-x} N_{x}$ on GaAs for long wavelength infrared applications

Journal of Applied Physics 111, 083104 (2012); 10.1063/1.3702453

Modeling of light scattering from micro- and nanotextured surfaces

Journal of Applied Physics 107, 044504 (2010); 10.1063/1.3295902

Optical modeling of a-Si:H solar cells deposited on textured glass $/ \mathrm{SnO}_{2}$ substrates

Journal of Applied Physics 92, 749 (2002); 10.1063/1.1487910

Light trapping properties of pyramidally textured surfaces

Journal of Applied Physics 62, 243 (1998); 10.1063/1.339189

\section{Scilight}

Sharp, quick summaries illuminating the latest physics research

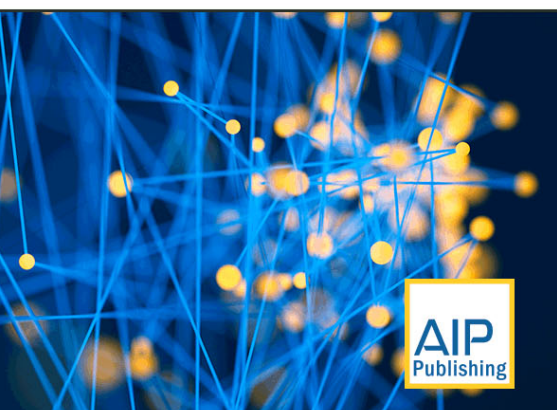




\title{
Light scattering at textured back contacts for $\mathbf{n}$-i-p thin-film silicon solar cells
}

\author{
K. Bittkau, W. Böttler, M. Ermes, V. Smirnov, and F. Finger \\ IEK5 - Photovoltaik, Forschungszentrum Jülich GmbH, 52425 Jülich, Germany
}

(Received 14 December 2011; accepted 9 March 2012; published online 16 April 2012)

\begin{abstract}
The angular resolved light scattering at textured back contacts for $n-i-p$ thin-film silicon solar cells is investigated experimentally in air. These results are compared to simulations performed by a scalar model for reflection with excellent agreement. Furthermore, light scattering is modeled for the transmission and reflection inside the silicon solar cell. It is found that the reflection at the back contact dominates the light scattering in the absorber layer. From these simulations, a quantity is derived that successfully predicts the external quantum efficiencies of solar cells on different textures. (C) 2012 American Institute of Physics. [http://dx.doi.org/10.1063/1.3703572]
\end{abstract}

\section{INTRODUCTION}

The theoretical understanding of light scattering at rough interfaces in thin-film silicon solar cells is of tremendous importance for the improvement of energy conversion efficiency. ${ }^{1-3}$ Due to the fact that the absorption length of light is longer than the thickness of the absorber layer for the long visible wavelength range and near infrared range, a large amount of light cannot be absorbed by a single path through the layer. Therefore, different concepts for a so-called light trapping can be applied. Typically, the light scattering at statistically rough interfaces is applied in thin-film solar cell technology. ${ }^{4}$

Commonly, the investigation of the scattering properties of such rough interfaces is done for textured transparent conductive oxides (TCOs) deposited on glass substrates in transmission geometry without silicon on top of the TCO. A typical quantity is the spectral haze which is the ratio of diffusely scattered light and the totally scattered light as a function of the wavelength. ${ }^{5}$ This quantity does not distinguish between light that is scattered into lower angles and light that is scattered into higher angles. Therefore, a more detailed characterization is often done by angular resolved light scattering (ARS) that measures the angular intensity distribution of the diffusely scattered light. ${ }^{6-8}$

Two main assumptions are made by using this characterization to evaluate the light trapping efficiency. First, the dominant light scattering process occurs at the transmission at the front interface of the solar cell. Second, the scattering into air with a lower refractive index than the TCO will lead to the same conclusions for effective light scattering as the light scattering into silicon with a higher refractive index. Both assumptions are daring, since at each rough interface between materials with different refractive indices scattering occurs for transmitted and reflected light. Therefore, other scattering processes than the transmission at the front contact should be taken into account. Moreover, the high refractive index of silicon leads to a higher optical mode density and it is possible that light modes can be excited which can propagate in silicon but not in air. ${ }^{9}$ These light modes are, therefore, trapped in the absorber layer. Since the trapped light can only be determined when silicon is present, the characterization of light scattering in air might give wrong conclusions regarding the light trapping potential of a given interface texture.
In this paper, the light scattering properties of differently textured back reflectors are investigated both in air and in silicon. On these back reflectors, hydrogenated microcrystalline silicon ( $\mu \mathrm{c}-\mathrm{Si}: \mathrm{H})$ solar cells are deposited in an n-i-p configuration. As a back reflector we use texture-etched $\mathrm{ZnO}: \mathrm{Al}$ covered with Ag. Variation of the $\mathrm{ZnO}$ :Al layer thickness combined with different etching times allow to realize and evaluate a much wider range of surface morphologies as if such structures would be used as front contact in solar cells with p-i-n configuration. The reason is that in the n-i-p configuration only the morphological properties of the $\mathrm{ZnO}: \mathrm{Al}$ layers are of importance while optical transparency and sheet resistance of the layer do not have to be considered. The experimental results for the light scattering in air are compared to simulations by using a scalar approach for reflection. Within the silicon, the light scattering is investigated both for the transmission at the front contact and the reflection at the back contact. It is found that the reflection at the back contact leads to much higher scattering angles. Assuming that the light trapping potential is defined by the amount of reflected light scattered into angles beyond the critical angle for total reflection, a very good agreement to measured external quantum efficiencies is found for a series of solar cells on different textures.

\section{EXPERIMENT}

The light scattering properties of the various reflectors are investigated with samples that consist of a $\mathrm{ZnO}$ :Al layer sputtered on a glass substrate. The surfaces of these layers are textured by wet chemical etching in $0.5 \mathrm{wt}$. \% hydrochloric acid. ${ }^{10}$ On top, Ag is deposited by thermal evaporation. The samples vary in the initial thickness of the $\mathrm{ZnO}$ :Al layer after sputtering and etching time. Both parameters have an influence on the resulting texture. The etching process reveals a crater-like surface roughness with statistically distributed lateral crater sizes and heights. These samples are subsequently used as back contacts for thin-film silicon solar cells in the $n-i-p$ configuration. Since the $\mathrm{ZnO}$ :Al layers investigated in this study only define the texture and are not part of the solar cell device, a wider range of surface morphologies can be compared than in a p-i-n configuration where the front contact layer of the solar cell is textured. 
In Fig. 1, the topographies measured by atomic force microscopy of three of the investigated samples are shown. In Fig. 1(a), a $50 \mathrm{~s}$ etched $\mathrm{ZnO}$ :Al layer with an initial thickness of $550 \mathrm{~nm}$ is plotted (sample I). Figures 1(b) and 1(c) show the surfaces of $10 \mathrm{~s}$ (sample II) and $60 \mathrm{~s}$ (sample III) etched $\mathrm{ZnO}: \mathrm{Al}$ layers, respectively, with an initial thickness of $1050 \mathrm{~nm}$. The surface of sample I shows some large craters that reach the glass substrate due to the thin $\mathrm{ZnO}: \mathrm{Al}$ layer. Despite this, the majority of the craters appear similar to those of sample II concerning the lateral sizes. Nevertheless, the typical height of the craters is larger for sample II, which means that the steepness angle increases due to the thicker $\mathrm{ZnO}: \mathrm{Al}$ layer. For sample III, the amount of larger craters is increased compared to samples I and II.

These samples are investigated by angular resolved scattering in reflection geometry. The schematic setup is shown in Fig. 2. The samples are illuminated with coherent light source at normal incidence and the diffusely scattered light is collected by a movable detector which can be rotated around the sample.

For each scattering angle $\theta$, the measured intensity $I(\theta)$ is multiplied by $\sin \theta$ to get the spherically integrated intensity. This results in the angular intensity distribution $\left(\operatorname{AID}_{\text {air }}(\theta)=I(\theta) \cdot \sin \theta\right)$ in air.

\section{SCATTERING MODEL FOR REFLECTION}

For the simulation of the light scattering, the so-called phase model is adapted to reflection geometry. This model was recently applied to textured $\mathrm{ZnO}: \mathrm{Al}$ films for the transmission into air and silicon ${ }^{11-13}$ and validated by experiment and rigorous solution of Maxwell's equations. In reflection geometry, the collected phase while traversing the roughness zone is given by

$$
\Delta \phi=\frac{2 \pi n}{\lambda} \cdot 2 \cdot\left(h_{\max }-h_{\min }-h(x, y)\right),
$$
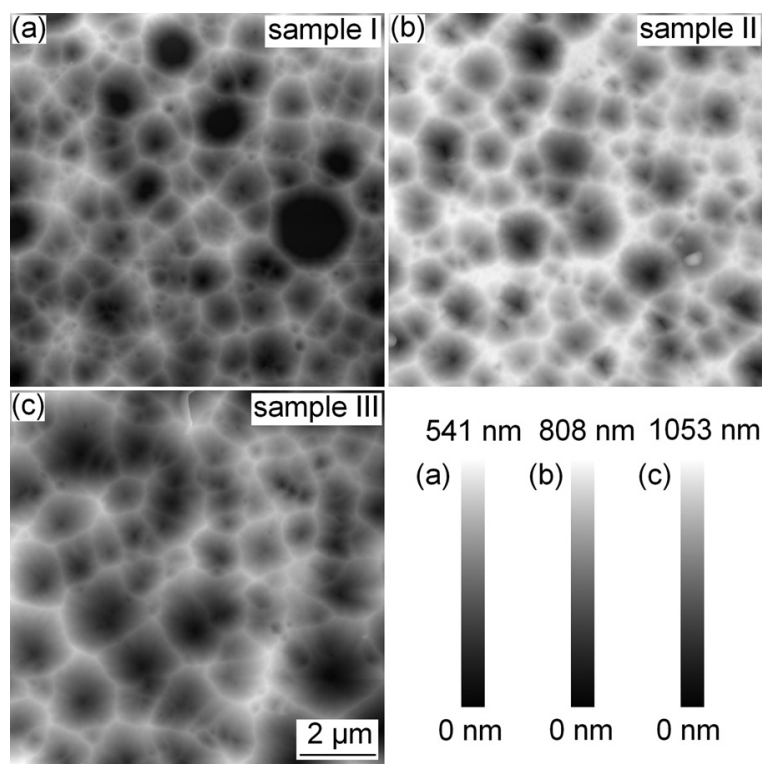

FIG. 1. Topographies of (a) $50 \mathrm{~s}$ etched $\mathrm{ZnO}: \mathrm{Al}$ with $550 \mathrm{~nm}$ initial thickness (sample I), (b) $10 \mathrm{~s}$ etched $\mathrm{ZnO}: \mathrm{Al}$ with $1050 \mathrm{~nm}$ initial thickness (sample II), and (c) $60 \mathrm{~s}$ etched $\mathrm{ZnO}: \mathrm{Al}$ with $1050 \mathrm{~nm}$ initial thickness (sample III) used as substrates for n-i-p solar cells.

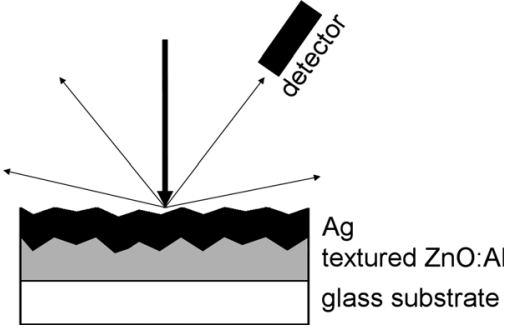

FIG. 2. Setup for angular resolved scattering in reflection geometry. The investigated samples consist of layer stacks of textured $\mathrm{ZnO}$ :Al on glass substrate. On top of the textured layer, an Ag layer is deposited.

with $h(x, y), h_{\max }, h_{\min }, n$, and $\lambda$ representing the topography of the surface, the largest and smallest height of the topography, the refractive index of the medium, and the wavelength in vacuo, respectively.

The scattered light intensity corresponds to the radiance, $\mathcal{L}$, which is defined as the radiant power per unit solid angle per unit projected source area. This quantity can be calculated by

$$
\mathcal{L}\left(k_{x}, k_{y}\right)=\frac{\lambda^{2}}{A_{S}}\left|\mathcal{F}\left\{\frac{1}{(1+\epsilon)^{2}} \exp (\mathrm{i} \Delta \phi)\right\}\right|^{2},
$$

where $A_{S}$ is the scattering surface, $\mathcal{F}\{\ldots\}$ is the Fourier transform, $k_{x}$ and $k_{y}$ are the $x$ and $y$ components of wave vector $k$, respectively, and $\epsilon$ is the ratio between the distance from the origin to all points $(x, y)$ of the integration and the observation distance. Since the upper limit of $\epsilon$ corresponds to the ratio between the diameter of the light beam and the observation distance, this value can be neglected.

For $k_{x}^{2}+k_{y}^{2} \leq k^{2}$, light is scattered into propagating modes, otherwise into evanescent modes. ${ }^{14}$ The latter do not contribute to far-field scattering properties. The AID is given by

$$
\operatorname{AID}(\theta)=\sum_{k_{x}^{2}+k_{y}^{2}=k^{2} \sin ^{2} \theta} \mathcal{L}\left(k_{x}, k_{y}\right) \cdot \frac{2 \pi n}{\lambda} \cos \theta .
$$

Note that $\lambda$ represents the wavelength in vacuo. The impact of the corresponding medium on the effective wavelength is already included by the refractive index $n$. Additionally it should be mentioned that a negative topography, which occurs when illuminating the interface from the other direction, will lead to the same AID in any cases. This is due to the fact that the terms with $h_{\max }-h_{\min }$ in Eq. (1) will lead to a constant factor in Eq. (2) which does not affect the results of a Fourier analysis. Therefore, a negative topography results in the complex conjugated function to be Fourier transformed. Since the square of the norm is determined in Eq. (2) for the radiance, the result will be the same.

\section{INVESTIGATION OF LIGHT SCATTERING IN AIR}

The phase model for reflection is applied to samples I-III and the simulated $\mathrm{AID}_{\text {air }}$ is compared to the measurement. For the simulations, the same topographies are assumed for the $\mathrm{Ag}$ surface as for the $\mathrm{ZnO}: \mathrm{Al}$ (see Fig. 1). The results are 


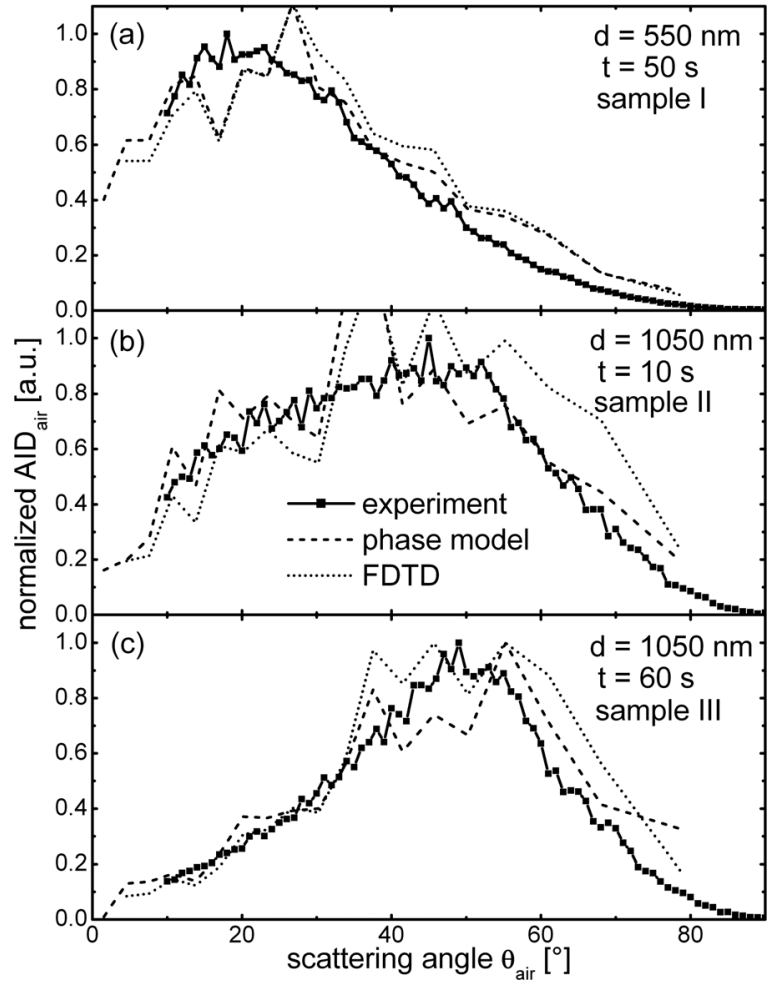

FIG. 3. Angular intensity distribution at a wavelength of $530 \mathrm{~nm}$ for sample I (a), sample II (b), and sample III (c) in air for the reflection at the rough Ag surface. Solid lines and symbols represent experimental results while dashed lines show results from the phase model. Simulated AIDs from FDTD are depicted as dotted lines.

depicted in Fig. 3 for a wavelength of $530 \mathrm{~nm}$. Each curve is normalized to its maximum for the sake of comparison.

$\mathrm{AID}_{\text {air }}$ varies significantly for the three samples. Sample I [Fig. 3(a)] shows a maximum scattering intensity at $20^{\circ}$ with a steeper slope for lower angles than for higher angles. Sample II [Fig. 3(b)] has a much broader AID $_{\text {air }}$ with a maximum at around $45^{\circ}$, while the maximum of the $\mathrm{AID}_{\text {air }}$ of sample III [Fig. 3(c)] is found at $50^{\circ}$ with a steeper slope for higher angles than for lower angles. In all cases, the agreement between the experimental results (solid lines and symbols) and the phase model (dashed lines) is very good. To validate the accuracy of the phase model, additional simulations are shown for a rigorous solution of Maxwell's equations using the finite-difference time-domain (FDTD) method (dotted lines in Fig. 3). ${ }^{15}$ Here, the same topographies as shown in Fig. 1 are considered. Due to the finite sampling size of the topographies, the angular resolution is reduced in the model as compared to the experiment. Additionally, in the experiment, a much larger area than the sampling size of $10 \mu \mathrm{m} \times 10 \mu \mathrm{m}$ is illuminated. Therefore, a much better statistical averaging of scatterers is achieved in the experiment than in the phase model. This leads to smoother shapes of the AIDs.

\section{INVESTIGATION OF LIGHT SCATTERING IN SILICON}

The investigation presented in Sec. IV was done for light scattering in air. However, the light scattering properties into silicon has to be studied to evaluate the light trapping potential of different textures. Regarding the layer stack of a thin-film silicon solar cell, two main scattering interfaces exist. First, the interface of the front contact $\mathrm{ZnO}: \mathrm{Al}$ and the p-layer of the $\mu \mathrm{c}-\mathrm{Si}: \mathrm{H}$ solar cell, where the transmitted light is scattered. Second, the back contact, where the reflected light is scattered. For both interfaces, the scattered light propagates inside silicon.

Within the phase model, the $\mathrm{AID}_{\mathrm{Si}}$ for the transmission at the $\mathrm{ZnO}: \mathrm{Al} / \mu \mathrm{c}-\mathrm{Si}: \mathrm{H}$ interface are simulated separately for sample I (solid line), sample II (dashed line), and sample III (dotted line) and are shown in Fig. 4 for a wavelength of $800 \mathrm{~nm}$. The ordinate represents the total amount of scattered light per angular element. The wavelength of $800 \mathrm{~nm}$ was chosen since at this wavelength the effects of light trapping in silicon are strongly pronounced. For the investigation in air, the scattering model was validated at a shorter wavelength since the total amount of scattered light is larger than at $800 \mathrm{~nm}$ and the light is scattered into smaller angles (not shown) which allows a wider angular range for the comparison.

Two important critical angles exists: (1) the total reflection angle for $\mu \mathrm{c}-\mathrm{Si}: \mathrm{H} /$ air and (2) for $\mu \mathrm{c}-\mathrm{Si}: \mathrm{H} / \mathrm{ZnO}: \mathrm{Al}$. The former leads to a total reflection at the front interface to air, the latter for light that is fully captured inside the absorber layer. Both critical angles are depicted as vertical lines in Fig. 4

The $\mathrm{AID}_{\mathrm{Si}}$ for transmission shows its maximum at around $8^{\circ}$ (sample I), $12^{\circ}$ (sample II), and $13^{\circ}$ (sample III), which is below the critical angle for $\mu \mathrm{c}-\mathrm{Si}: \mathrm{H} /$ air $\left(15.8^{\circ}\right)$ and significantly below that for $\mu \mathrm{c}-\mathrm{Si}: \mathrm{H} / \mathrm{ZnO}: \mathrm{Al}\left(26.5^{\circ}\right)$.

For comparison, the $\mathrm{AID}_{\mathrm{Si}}$ for the reflection in silicon at the back contact is modeled for the different textures for a wavelength of $800 \mathrm{~nm}$. It is assumed that the light irradiates perpendicular to the surface. This assumption allows the evaluation of the direct light scattering at the back reflector. It is not the focus of this paper to study the dependence of the light scattering properties on the angle of incidence. It will be shown that the light is scattered into much higher angles at the back reflector than at the front contact, which makes the assumption reasonable that the scattering at the front contact has only a minor impact on the light trapping in the solar cell.

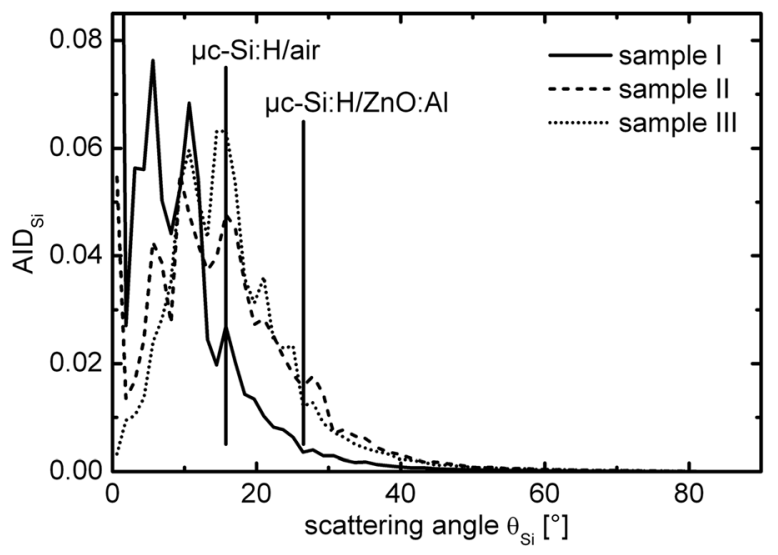

FIG. 4. Angular intensity distribution at a wavelength of $800 \mathrm{~nm}$ in silicon for the transmission at the front contact for three different samples. Sample I is shown as a solid line, sample II as a dashed line, and sample III as a dotted line. The critical angles for total reflection at the $\mu \mathrm{c}-\mathrm{Si}: \mathrm{H} / \mathrm{air}$ and $\mu \mathrm{c}-\mathrm{Si}: \mathrm{H} / \mathrm{ZnO}: \mathrm{Al}$ interface are depicted as vertical lines. 


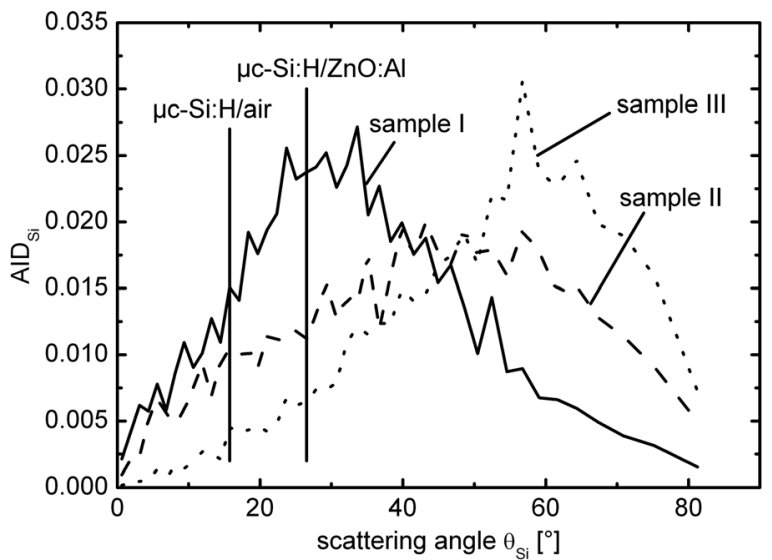

FIG. 5. Angular intensity distribution at a wavelength of $800 \mathrm{~nm}$ in silicon for the reflection at the back contact for three different samples. Sample I is shown as a solid line, sample II as a dashed line, and sample III as a dotted line. The critical angles for total reflection at the $\mu \mathrm{c}-\mathrm{Si}: \mathrm{H} / \mathrm{air}$ and $\mu \mathrm{c}-\mathrm{Si}: \mathrm{H} /$ $\mathrm{ZnO}: \mathrm{Al}$ interface are depicted as vertical lines.

The $\mathrm{AID}_{\mathrm{Si}}$ in reflection is exemplarily shown for samples I-III in Fig. 5. Again, the total amount of scattered light per angular element is plotted against the scattering angle. Both critical angles are depicted by vertical lines. Sample I has its maximum in the $\mathrm{AID}_{\mathrm{Si}}$ at around $30^{\circ}$ (solid line), whereas sample II (dashed line) and III (dotted line) show the highest scattering intensity at around $50^{\circ}$ and $60^{\circ}$, respectively. As for the reflection in air, the broadest angular distribution is found for sample II. For all three samples, the maximum is beyond the critical angle for $\mu \mathrm{c}-\mathrm{Si}: \mathrm{H} / \mathrm{ZnO}: \mathrm{Al}$. However, sample III is found to show the largest amount of light that is scattered beyond this critical angle.

Compared to the light scattering in transmission at the front contact, where all maxima in the intensity are found for angles below the critical angles, it can be concluded that the dominant scattering process regarding the light trapping occurs at the textured back contact. Moreover, the differences between the various textures are much more pronounced.

\section{COMPARISON TO SOLAR CELL PROPERTIES}

For a series of textured back reflectors, $\mu \mathrm{c}-\mathrm{Si}: \mathrm{H}$ solar cells are prepared in an n-i-p configuration with sputtered $\mathrm{ZnO}: \mathrm{Al} / \mathrm{Ag}$ back reflectors. ${ }^{16}$ These back reflectors are deposited on textured $\mathrm{ZnO}$ :Al layers with initial thicknesses of 550,800 , and $1050 \mathrm{~nm}$ and with various etching times for each thickness. The $\mathrm{ZnO}$ :Al layers on top of the Ag have a thickness of $80 \mathrm{~nm}$. The silicon deposition was done by plasma-enhanced chemical vapor deposition successively under reproducible conditions to have a set of comparable cells. All cells are characterized by measuring the external quantum efficiency. The results are shown for four samples in Fig. 6(a). The surface of the sample with $800 \mathrm{~nm}$ initial $\mathrm{ZnO}: \mathrm{Al}$ thickness and an etching time of $6 \mathrm{~s}$ exhibits a comparably flat shape which results in pronounced interference fringes in the quantum efficiency in the long wavelength range. Weaker interference effects are also found for other samples. Since the phase model does not take these interferences into account, the measured quantum efficiencies are smoothed for comparison with simulations. These smoothed

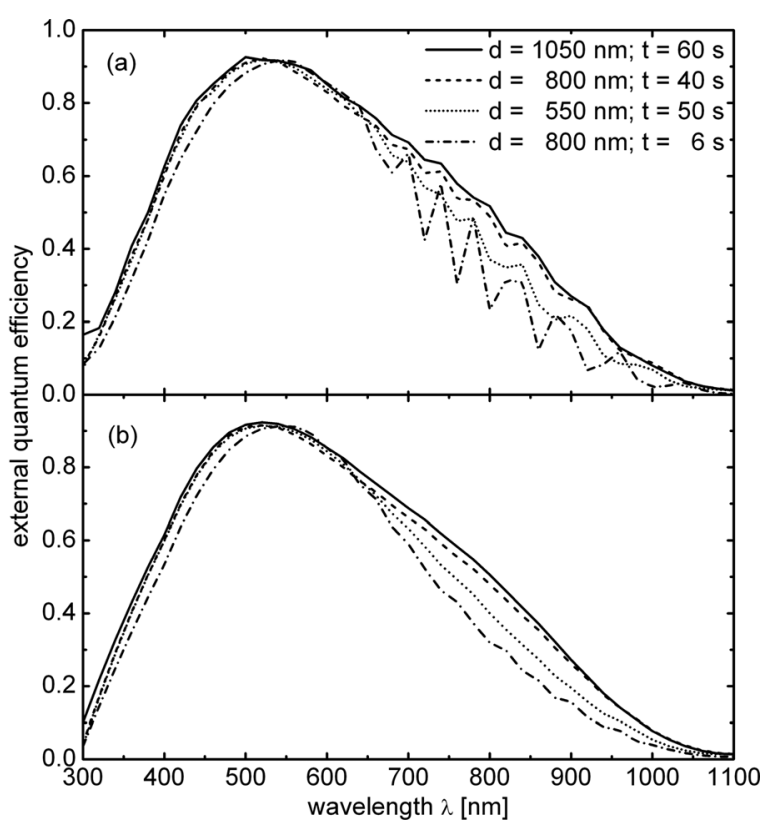

FIG. 6. External quantum efficiency of four of the investigated samples. The solid lines represent the result for the sample with $1050 \mathrm{~nm}$ initial $\mathrm{ZnO}$ :Al thickness and an etching time of $60 \mathrm{~s}$ (sample III). Sample I (initial thickness of $550 \mathrm{~nm}$ and etching time of $50 \mathrm{~s}$ ) is shown as a dotted line. Additionally, the results for cells deposited on textured $\mathrm{ZnO}: \mathrm{Al}$ with initial thickness of $800 \mathrm{~nm}$ and etching times of 40 and $6 \mathrm{~s}$ are shown as dashed and dashed-dotted lines, respectively. In (a) the direct measured results are depicted. To avoid influences of the interference fringes for the comparison with the phase model, the measured quantum efficiencies are smoothed, resulting in the curves shown in (b).

curves are shown in Fig. 6(b). In the long wavelength range, strong variations are found for the different samples. The values at a wavelength of $800 \mathrm{~nm}$ are taken for the comparison with the light trapping potential predicted by the phase model.

Figure 7 shows the smoothed quantum efficiency from the experiment at a wavelength of $800 \mathrm{~nm}$ plotted against the amount of scattered light with angles larger than the critical angle for total reflection at the $\mu \mathrm{c}-\mathrm{Si}: \mathrm{H} / \mathrm{ZnO}: \mathrm{Al}$ interface. The latter quantity is assumed to be a measure for the light trapping potential since total reflection leads to guided optical modes in the absorber layer. ${ }^{17}$

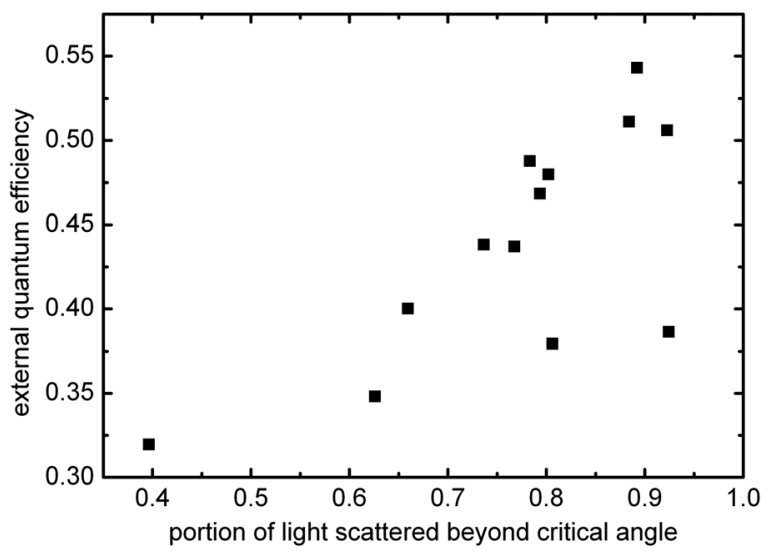

FIG. 7. External quantum efficiency at a wavelength of $800 \mathrm{~nm}$ plotted against predicted light trapping potential calculated from the phase model for solar cells on different textured substrates. Each point represents one sample. 
It is found that the external quantum efficiency is strongly defined by the amount of light that is scattered beyond the critical angle at the back reflector. This amount varies from 0.4 in the case of the sample with $800 \mathrm{~nm}$ initial $\mathrm{ZnO}: \mathrm{Al}$ thickness and an etching time of $6 \mathrm{~s}$ to 0.92 for the sample with $1050 \mathrm{~nm}$ initial $\mathrm{ZnO}: \mathrm{Al}$ thickness and an etching time of $60 \mathrm{~s}$. The latter means that more than $90 \%$ of the light scattered at the back reflector is scattered sufficiently for light trapping by one single scattering process, whereas for the former sample this portion is only $40 \%$. This leads to the strong variation in the quantum efficiency from 0.32 to 0.51 for these two samples.

This shows that the dominant light scattering process regarding the light trapping occurs at the back reflector since from the $\mathrm{AID}_{\mathrm{Si}}$ for this reflection, the quantum efficiency of the whole cell can be predicted. Other positive optical effects of the texture, such as the gradual matching of the refractive indices that leads to a reduction of spurious reflection, occur at the front contact and affects the whole spectrum, particularly the short wavelength range. This effect can also be seen in Fig. 6 for wavelengths below $500 \mathrm{~nm}$. Here, the sample with $800 \mathrm{~nm}$ initial $\mathrm{ZnO}$ :Al thickness and etching time of $6 \mathrm{~s}$ shows significantly lower quantum efficiencies due to the comparably flat surface.

\section{CONCLUSION}

Light scattering properties at different textured back reflectors are investigated by angular resolved scattering. Results from simulations by the phase model agree well with experiment. For the light scattering inside the solar cell, the model is applied to silicon for the transmission at the front contact as well as for the reflection at the back contact. It is found that the reflection at the back contact leads to much higher scattering angles than the transmission at the front contact. Therefore, it is concluded that the back reflector plays a dominant role for an effective light trapping inside the absorber layer. The amount of light that is scattered into angles beyond the critical angle for total reflection exceeds $90 \%$ and shows good correlation to experimentally determined quantum efficiencies of the solar cells. Therefore, this model can be used to predict the light trapping potential in $\mu \mathrm{c}-\mathrm{Si}: \mathrm{H}$ solar cells.

\section{ACKNOWLEDGMENTS}

Uwe Rau is gratefully acknowledged for continuous support and encouragement.

${ }^{1}$ E. Yablonovitch, J. Opt. Soc. Am. 72, 899 (1982).

${ }^{2}$ P. Obermeyer, C. Haase, and H. Stiebig, Appl. Phys. Lett. 92, 181102 (2008).

${ }^{3}$ C. Rockstuhl, S. Fahr, F. Lederer, K. Bittkau, T. Beckers, and R. Carius, Appl. Phys. Lett. 93, 061105 (2008).

${ }^{4}$ H. W. Deckman, C. R. Wronski, H. Witzke, and E. Yablonovitch, Appl. Phys. Lett. 42, 968 (1983).

${ }^{5}$ O. Kluth, B. Rech, L. Houben, S. Wieder, G. Schöpe, C. Beneking, H. Wagner, A. Löffl, and H. W. Schock, Thin Solid Films 351, 247 (1999).

${ }^{6}$ H. Schade and Z. E. Smith, Appl. Opt. 24, 3221 (1985).

${ }^{7}$ J. Krč, M. Zeman, O. Kluth, F. Smole, and M. Topič, Thin Solid Films 426, 296 (2003).

${ }^{8}$ K. Jäger, O. Isabella, R. A. C. M. M. van Swaaij, and M. Zeman, Meas. Sci. Technol. 22, 105601 (2011).

${ }^{9}$ K. Bittkau and T. Beckers, Phys. Status Solidi A 207, 661 (2010).

${ }^{10}$ M. Berginski, J. Hüpkes, M. Schulte, G. Schöpe, H. Stiebig, B. Rech, and M. Wuttig, J. Appl. Phys. 101, 074903 (2007).

${ }^{11}$ D. Dominé, F.-J. Haug, C. Battaglia, and C. Ballif, J. Appl. Phys. 107, 044504 (2010).

${ }^{12}$ K. Bittkau, M. Schulte, M. Klein, T. Beckers, and R. Carius, Thin Solid Films 519, 6538 (2011).

${ }^{13}$ M. Schulte, K. Bittkau, K. Jäger, M. Ermes, M. Zeman, and B. E. Pieters, Appl. Phys. Lett. 99, 111107 (2011).

${ }^{14}$ J. Harvey, Am. J. Phys. 47, 974 (1979).

${ }^{15}$ A. F. Oskooi, D. Roundy, M. Ibanescu, P. Bermel, J. D. Joannopoulos, and S. G. Johnson, Comput. Phys. Commun. 181, 687 (2010).

${ }^{16}$ W. Böttler, V. Smirnov, A. Lambertz, J. Hüpkes, and F. Finger, Phys. Status Solidi C 7, 1069 (2010).

${ }^{17}$ A. Banerjee and S. Guha, J. Appl. Phys. 69, 1030 (1990). 